\title{
A SEARCH FOR MASSIVE COMPACT HALO OBJECTS IN OUR GALAXY
}

\author{
presented by D. P. Bennett
}

in collaboration with C. Alcock, T. Axelrod, K. Cook, H. Park Lawrence Livermore National Laboratory, Livermore, CA, USA

K. Griest, C. Stubbs

Center for Particle Astrophysics, U.C. Berkeley, Berkeley, CA, USA

K. Freeman, B. Peterson, P. Quinn, A. Rodgers

Mount Stromlo and Siding Spring Observatories, Canberra, Australia

November 16, 1990

\section{Abstract}

MAssive Compact Halo Objects such as brown dwarfs, Jupiters, and black holes are prime candidates to comprise the dark halo of our galaxy. Paczynski noted that these objects (dubbed MACHOs) can be detected via gravitational microlensing of stars in the Magellanic Clouds with the caveat that only about one in $10^{6}$ stars will be lensed at any given time. Our group is currently involved in constructing a dedicated observing system at the Mount Stromlo Observatory in Australia. We will use a refurbished 1.27 meter telescope and an innovative two-color CCD camera with $3.4 \times 10^{7}$ pixels to monitor $10^{6}-10^{7}$ stars in the Magellanic Clouds. During the first year of operation (1991-1992), we hope to detect (or rule out) objects in the mass range $0.001 M_{\odot} \leq M \leq 0.1 M_{\odot}$, and after five years, we hope to have covered the range $10^{-6} M_{\odot}<M \lesssim 100 M_{\odot}$.

\section{MICROLENSING BASICS}

Gravitational microlensing refers to lensing in the case where there is significant amplification of the source, but the lensing angle is too small to be observed. Paczynski ${ }^{1}$ has shown that the "optical depth" for microlensing by the dark halo of our galaxy is about $10^{-6}$, so that at any given time about 1 star in $10^{6}$ will be microlensed with amplification by a factor of 1.34 or larger. (The typical angular separation of the images is $\lesssim 0.001$ arc second, so they cannot be resolved.) The fundamental unit of length for a microlensing 
is the Einstein ring radius which is given by

$$
R=2\left(\frac{G M x(L-x)}{c^{2} L}\right)^{1 / 2}
$$

where $L$ is the distance to the source star (in our case in the Magellanic Clouds) and $x$ is the distance to the lensing object in the halo. If $R$ is not too small, the source star can be assumed to be a point light source, and the microlensing amplification depends only on the dimensionless impact parameter ${ }^{1-3}(u \equiv b / R)$ :

$$
A(u)=\frac{u^{2}+2}{u \sqrt{u^{2}+4}}
$$

$u=1$ corresponds to an amplification of 1.34 .

The time scale of a microlensing event is given by the timescale for the lensing object to traverse a distance equal to the impact parameter $b$ which yields

$$
\Delta t=7 \text { days } \sqrt{\frac{M}{10^{-2} M_{\odot}}}
$$

for a typical lensing object located at a distance of $10 \mathrm{kpc}$ moving at a velocity of 200 $\mathrm{km} / \mathrm{sec}$.

The microlensing light curves will be distinguishable from the background of variable stars in several important ways: they are achromatic, time-symmetric, and non-repeating. These signatures should allow us to distinguish most (and hopefully all) of the variable stars from the microlensing events. In addition, the microlensing light curve is described by just 3 parameters: the maximum amplification, the time of the maximum amplification and the duration of the event. Clear evidence of this characteristic light curve will be required in order to distinguish microlensing by MACHOs from other phenomena such as variable stars.

There are several possible complications which might tend interfere with the characteristic microlensing light curve. First, the finite size of the source star adds another pararneter to the microlensing lightcurve, but for halo objects with $M>10^{-4} M_{\odot}$, most of the red giants in the Magellanic Clouds would appear as point-like sources. Another potential complication comes from double stars. These would add another parameter to the lightcurve fit and could make the microlensing events appear chromatic, but we have found that most double star light curves can be fit very well by a single star light curve. The only real problem would be the different light curves observed in the two color bands when a member of a red giant-main sequence pair is microlensed. Fortunately, red giants are bright only for a very short portion of their lifetime, so the probability that a red giant will be paired with a main sequence companion of comparable brightness is small. One might also worry that the microlensing of double stars might be more difficult to detect because apparent amplitude of the lightcurve will be reduced when only one member of the pair is microlensed. This is a very small effect, however, because the second member of the pair can also be lensed, and this possibility mostly compensates for the number of events lost due to the presence of double stars. 


\section{THE TELESCOPE}

We plan to carry out our microlensing search on the Great Melbourne (50") Telescope at Mount Stromlo which is being refurbished for dedicated use in our microlensing search. This telescope is an "English" (two-pier, crossed axis) mounted f 4.47 reflector of $1.27 \mathrm{~m}$ aperture and $5.67 \mathrm{~m}$ focal length. We will install our field corrector and double CCD camera package at the prime focus. The corrector package will reduce the effective focal length to $4.95 \mathrm{~m}$ ( $\mathrm{f} 3.9$ ) and provide a usable field of greater than $8.7 \mathrm{~cm}=60$ arc minutes in diameter.

The main advantages of this telescope are the proximity to our collaborators in Australia and high degree of support from MSSSO. The Australian telescope will also be ready to observe starting in the 1991-1992 Magellanic cloud season, which is significantly earlier than a new telescope could be made ready. The main disadvantages of the telescope are the weather and seeing conditions. The telescope site is completely overcast about $35 \%$ of the nights and completely clear on about $35 \%$ of the nights. The remaining $30 \%$ of the nights have some useable time. The median seeing is about 1.6" FWHM. We have analyzed both of these difficulties, and found that they should not prevent us from detecting or ruling out the dark matter in the mass range that we plan to probe.

\section{THE CAMERA}

The most innovative part of our experiment is our CCD camera. We plan to observe simultaneously in two different color bands. (The first generation camera will use color bands that are approximately equal to the Gunn $g$ band and the Johnson $R$ band.) This will be accomplished by splitting the beam with a glass cube dichroic element which will send the beams to separate $2 \times 2$ mosaics of $2 \mathrm{k} \times 2 \mathrm{k}$ Ford Aerospace edge butable CCDs.

\section{OBSERVATIONAL STRATEGY}

Our precise observational strategy has been by no means decided yet, but the following seems to be the most reasonable strategy at present. We will survey stars in the LMC, the SMC and the galactic bulge, and we plan to work to a limiting magnitude of about $m<19.5$ with 5 minute exposures (at full moon). We tentatively plan to work out to a radius of about 3 degrees from the center of the LMC and 2 degrees from the center of the SMC. (Both these numbers refer to the distance along the major axis.) The estimated star densities $(m<19.5)$ in these fields range from about 380,000 stars per square degree in the center of the LMC to about 85,000 stars per square degree 3 degrees away from the center of the LMC to 30,000 stars 2 degrees frorn the center of the SMC. An analysis of existing CCD frames of a region in the Magellanic Clouds taken in different seeing conditions using standard crowded field photometry software has indicated that we should be able to get accurate (better than 5\%) photometry on 120,000 stars per square degree with 3 " seeing and on $>200,000$ stars per square degree with 1.5 " seeing. This means that we should be able to get about 2,000,000 stars in a night with 3 " seeing in the prime cloud season 
and 2,500,000 in a nigh: with 1.5" seeing. During a new moon, we should be able to get about $3,200,000$ stars on a good night.

We plan to concentrate initially on the mass range $10^{-3} M_{\odot} \leq M \leq 10^{-1} M_{\odot}$ which spans the range for Jupiters and brown dwarfs. The time scale for these events ranges from a couple days to a few weeks, so a reasonable strategy will be to observe as many different stars as possible each night. As many of the same stars as possible will be observed on successive nights so as to give daily sampling of the light curves for as many stars as possible.

In order to search for halo objects of larger mass (black holes?), it will be preferable to sample more stars less frequently. One relatively easy way to do this will be to co-add data from successive nights in order to get accurate photometry on fainter stars. In order to get data on the $10^{7}$ stars which would be required to be able to detect microlensing by objects of $100 M_{\odot}$ or more, we will probably have to take longer exposures in some fields that are further from the centers of the clouds.

Smaller mass dark matter candidates $\left(M \leq 10^{-4} M_{\odot}\right)$ pose several different problems. First, the lensing light curves for red giants (which make up two thirds of the stars to $m<$ 19.5) in the clouds become more complicated because the point-like source approximation begins to fail. For impact parameters comparable to the stellar radius, this results in a large increase in the amplification, but when the impact parameter is smaller than the stellar radius, the amplification is reduced. The other difficulty with these small mass objects is that they tend to have lightcurves which last about 24 hours which makes it difficult to obtain complete light curves. The detection of microlensing in this mass range will probably require the use of at least two different observing sites at different longitude.

\section{PREDICTED EVENT RATES}

We have examined 9 years of detailed weather records to come up with the following event rates. The Halo was assumed to consist of a Halo density profile of the form ${ }^{2}$

$$
\rho=\rho_{0} \frac{a^{2}+r_{0}^{2}}{a^{2}+r^{2}}
$$

where the core radius $a=5 \mathrm{kpc}$ and the local Halo density $\rho_{0}=0.0079 M_{\odot} \mathrm{pc}^{-3}$. The table shows the average number of events detected using 8 years of weather data from Mt. Stromlo for different masses of Halo objects. The two detection criteria used are (1) to require 2 points with $A>1.2$ on each of the rising and falling parts of the light curve for a total of at least four points on the light curve with $A>1.2$, and (2) at least 5 points on the light curve with at least 2 on the rising and falling portions of the curve and 1 point with $A>2$. Note that each "point" on the light curve refers to 2 simultanenus images in different color bands. 


\begin{tabular}{|l|l|l|}
\hline Mass & 4 pts. with $A>1.2$ & $\begin{array}{l}5 \text { pts. with } A>1.2 \\
\& 1 \text { with } A>2\end{array}$ \\
\hline $10^{-4} M_{\odot}$ & 2.3 events/yr. & 0.8 events/yr. \\
$3 \times 10^{-4} M_{\odot}$ & 7.2 events/yr. & 2.8 events/yr. \\
$10^{-3} M_{\odot}$ & 15.9 events/yr. & 6.6 events/yr. \\
$3 \times 10^{-3} M_{\odot}$ & 23.7 events/yr. & 10.8 events/yr. \\
$10^{-2} M_{\odot}$ & 24.2 events/yr. & 11.4 events/yr. \\
$3 \times 10^{-2} M_{\odot}$ & 19.3 events/yr. & 8.9 events/yr. \\
$10^{-1} M_{\odot}$ & 12.8 events/yr. & 5.7 events/yr. \\
\hline
\end{tabular}

\section{THE USE OF ADDITIONAL SITES}

For Halo objects of $M \lesssim 10^{-4} M_{\odot}$, it is highly desirable to be able to track microlensing events with points evenly spaced at intervals of 2-10 hours. At most southern Hemisphere observing sites, the clouds can rarely be observed for much more than 8 hours in a single night, it may be necessary to use different telescopes a, different longitudes to sample this mass range. One option that may become available is to collaborate with the French group ${ }^{4}$ which will search for microlensing from a $40 \mathrm{~cm}$ refracting telescope at ESO. Another option would be to develop an alert mechanism that would allow follow up observations of ongoing microlensing events at other telescopes.

\section{ACKNOWLEDGEMENTS}

This work was supported in part by the U.S. Department of Energy by Lawrence Livermore National Laboratory under contract No. W-7405-Eng-48 and by the office of Science and Technology Centers of the NSF, under cooperative agreement AST-8809616.

\section{REFERENCES}

[1] B. Paczynski, Ap. J. 304, 1 (1986).

[2] K. Griest, Ap. J., in press, (1990).

[3] R. Nemiroff, NRL preprint, (1990).

[4] A. Milsztajn, to appear in the proceedings of the 10th Moriond Workshop on new and exotic phenomena, Les Arcs, France, 1990.

\section{DISCUSSION:}

Nemiroff: What technical advances in the last four years make this experiment feasible now?

Bennett: It is not clear that this experiment couldn't have been performed four years ago when Paczynski first proposed it nor is it clear that it can be done now. The main difficulty with this experiment is the "background events" caused by variable stars, and it will only be when these background events are understood will it be clear what technology is necessary for this experiment. Our approach makes use of the recently developed largeformat CCDs in order to do accurate photometry on a large number of stars, but the French approach is to obtain the photometry from Schmidt plates. If this is successful, then one might argue that this experiment could have been done four years ago. 
This document was prepared as an account of work sponaored by an agency of the United States Goverament. Neither the United States Government nor the University of Californin nor any of their employees, makes any warranty, express or implied, or assumes any legal liability or responsibility for the accuracy, completenesa, or usefulness of any information. apparatus, product, or process disclosed, or represents that its use would not infringe privately owned rights. Reference herein to any specific conimercial products, process, or service by trade name, trademark, manufacturer, or otherwise, does not necessarily constitute or imply its endorsement, recommendation, or tavoring by the United States Government or the University of Californin. The views and opiniosis of authors expressed herein do not necessarily state or reflect those of the United States Government or the University of California, and shall not be used for advertising or product endorsement purposes.

This is a preprint of a paper intended for publication in a journal or proceedings. Since changes may be made before publication, this preprint is made available with the understanding that it will not be cited or reproduced without the permission of the author. 

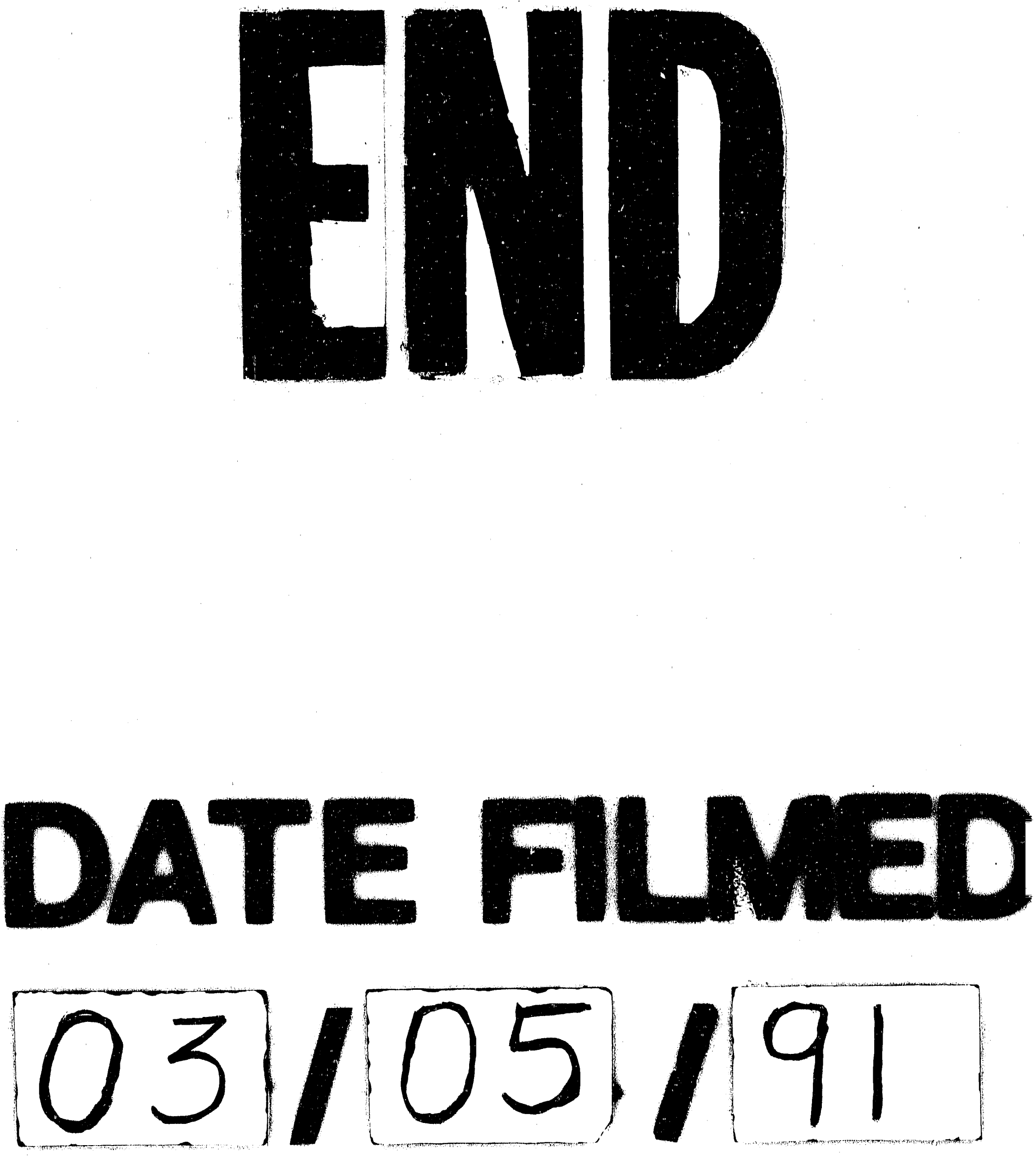
